\title{
ciência
}

\section{DETERMINANTES SOCIOECONÔMICOS DA SAÚDE DO IDOSO: UM ESTUDO ECOLÓGICO NO ESTADO DO RIO GRANDE DO NORTE}

\section{Socioeconomic determinants of elderly health: an ecological study in Rio Grande do Norte state}

Tamires Carneiro de Oliveira • Odontóloga. Doutoranda do Programa de Pós-graduação em Saúde Coletiva, Universidade Federal do Rio Grande do Norte. E-mail: tamires.carneiro@hotmail.com

Marla Raquel Leandro Silva • Graduanda de Odontologia. Universidade Federal do Rio Grande do Norte. E-mail: marlalleandro@gmail.com

Yan Nogueira Leite de Freitas • Doutorando do Programa de Pós-graduação em Saúde Coletiva, Universidade Federal do Rio Grande do Norte. E-mail: nlfyan@hotmail.com

André Luiz Lima - Doutor em Saúde Coletiva, Universidade Federal do Rio Grande do Norte. E-mail: andreveterinario@hotmail.com

Kenio Costa de Lima • Doutor em Ciências (Microbiologia), Professor adjunto do Departamento de Odontologia, Universidade Federal do Rio Grande do Norte. E-mail: limke@hotmail.com

Autora responsável pela correspondência:

Tamires Carneiro de Oliveira. Odontóloga. Doutoranda do Programa de Pós-graduação em Saúde Coletiva, Universidade Federal do Rio Grande do Norte. E-mail: tamires.carneiro@hotmail.com 


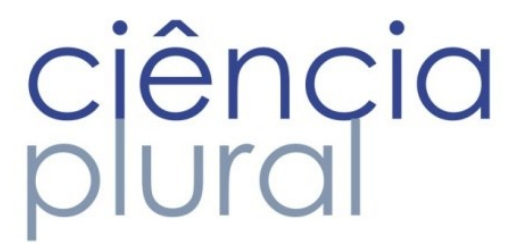

RESUMO

Objetivos: Propor variáveis estatísticas que resumam os fatores socioeconômicos com potencial efeito sobre a saúde dos idosos nos municípios do Rio Grande do Norte e identificar os locais de maior vulnerabilidade. Métodos: As variáveis deste estudo ecológico foram oriundas do Instituto Brasileiro de Geografia e Estatística - IBGE, Programa das Nações Unidas para o Desenvolvimento - PNUD, Instituto de Pesquisa Econômica Aplicada - IPEA e Departamento de Informática do SUS - DATASUS, referentes ao ano 2000. Partiu-se de mais de 800 variáveis relacionadas à renda, educação, desenvolvimento humano, demografia e habitação, as quais foram submetidas a uma análise da plausibilidade teórica, da distribuição dos dados, da correlação, Análise Fatorial e à Análise Espacial exploratória. Resultados: A aplicabilidade da técnica foi comprovada pela matriz de correlações, determinante $(0,001)$, matriz anti-imagem, teste de esfericidade de Bartlett $(p<0,001)$ e Índice de Kaiser-Meyer-Olkin $(\mathrm{KMO}=0,7)$. Formaram-se quatro componentes que explicaram $74,01 \%$ da variância do modelo, sendo eles: Melhores condições socioeconômicas, Maior dependência do Estado, Desigualdade e Menos emprego e saúde. A análise visual dos mapas demonstrou que tais componentes apresentam distintos padrões de distribuição espacial, no qual parte das microrregiões Borborema Potiguar, Angicos e Agreste Potiguar tiveram características mais desfavoráveis para todos os componentes. Conclusões: Além de facilitar a identificação dos pontos críticos, os componentes agregaram diversos dados em informações mais simples, facilitando o planejamento de políticas públicas e estudos relacionados aos determinantes da saúde dos idosos do Rio Grande do Norte.

Palavras-chave: Idosos; Fatores Socioeconômicos; Análise Fatorial.

\section{ABSTRACT}

Objective: To propose statistical variables that resume the socioeconomic factors with potential effect on elderly health in the municipalities of Rio Grande do Norte and to identify the most vulnerable places. Methods: The variables of this ecological study were collected from the Instituto Brasileiro de Geografia e Estatística - IBGE, Programa das Nações Unidas para o Desenvolvimento - PNUD, Instituto de Pesquisa Econômica Aplicada - IPEA and Departamento de Informática do SUS - DATASUS, about the year 2000. We started with more than 800 variables related to income, education, human development, demography and housing. These variables were submitted to theoretical plausibility analysis, data distribution, correlation, Factorial Anlysis and Exploratory Spatial Analysis. Results: The applicability of the technique was confirmed by the correlation matrix, determinant $(0,001)$, anti-image matrix, Bartlett's sphericity test and Kaiser-MeyerOlkin index $(\mathrm{KMO}=0,7)$. Four components were formed, which explained $74,01 \%$ of the model variance, they are: Better socioeconomic conditions, Greater government dependence, Inequity and Less employment and health. The visual analysis of the maps showed that these components have different patterns of spatial distribution, in which part of Borborema Potiguar, Angicos and Agreste Potiguar microregions had more unfavorable characteristics for all the components. Conclusions: Besides facilitating the identification of critical points, the components gathered several data in simpler information, helping in the planning of public policies and studies related to determinants of elderly health in Rio Grande do Norte.

Key words: Elderly; Socioeconomic Factors; Statistical Factor Analysis. 


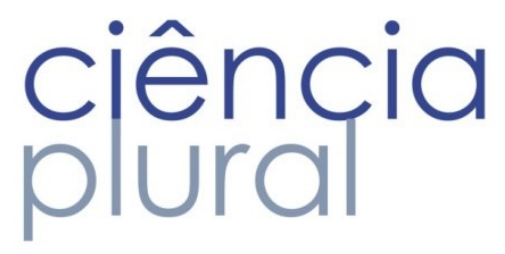

\section{Introdução}

O acelerado processo de envelhecimento populacional vivido pelo Brasil, com um crescimento de aproximadamente $700 \%$ no número de idosos no decorrer dos últimos 50 anos, traz uma série de desafios para gestores e pesquisadores dos sistemas de saúde, com repercussões para a sociedade como um todo. Ao contrário dos países desenvolvidos, que elevaram sua renda e implementaram estratégias para 0 desenvolvimento social previamente ao processo de envelhecimento, o Brasil se depara com as demandas de uma sociedade que envelhece em um contexto de acentuada desigualdade social, pobreza e fragilidade das instituições ${ }^{1}$.

Nesse contexto, a região Nordeste do Brasil se destaca por sua desvantagem em relação às outras regiões, de modo que essa, historicamente, não conseguia se desenvolver a ponto de proporcionar uma reprodução ampliada do seu capital2 2 . O desenvolvimento técnico e econômico do território brasileiro ocorreu de forma desordenada, seguindo um padrão de concentração geográfica no qual predominaram o acúmulo de recursos em determinadas áreas, as chamadas Regiões Concentradas do Brasil ${ }^{3}$. Em vista disso, a porção centralizada no Sul e Sudeste do país deteve um desenvolvimento industrial e financeiro mais expressivo que outras áreas, como o Nordeste, o que resultou em uma obtenção de sistemas de saúde, educação e lazer mais eficazes e que se perpetuam.

Tal situação ilustra o papel do território como eixo de iniquidade em saúde. Os fatores socioeconômicos se expressam como um dos principais determinantes das condições de vida de um indivíduo, de maneira a destacar a possibilidade do acesso a variados alimentos, à habitação, à educação, entre outros aspectos que corroboram para a construção de um estado de saúde. Assim, diante da existência de iniquidades, que se referem às injustas diferenças de oportunidades e recursos, em grande parte relacionadas à classe social, sexo, etnia ou território, produzem-se as desigualdades em saúde. ${ }^{4}$ De maneira significativa, as piores condições de saúde são frequentemente encontradas nos grupos socialmente menos favorecidos, especialmente para a população idosa, que apresenta características de exclusão social e também expressam o efeito cumulativo do constructo socioeconômico ao longo da vida 5 .

O estudo dos fatores socioeconômicos que abrangem a população idosa, para destacar os territórios de maior vulnerabilidade e os fatores associados ao mesmo, pode orientar o planejamento de políticas públicas que atendam a essa demanda populacional. Existem hoje bancos de dados de domínio público que disponibilizam uma ampla quantidade de variáveis socioeconômicas, entretanto, apesar de tais dados 


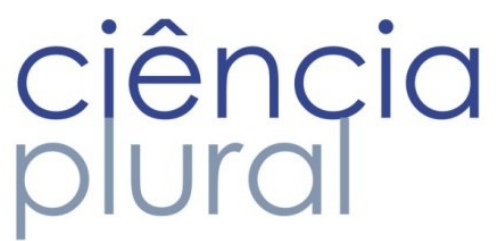

fornecerem um robusto subsídio para a avaliação e planejamento de políticas públicas, o grande volume de informações dificulta a análise deste contexto socioeconômico com um todo.

Desta forma, o presente trabalho tem por objetivo sintetizar os fatores socioeconômicos contextuais oriundos de fontes secundárias para 0 ano 2000 , com potencial efeito sobre a saúde dos idosos nos municípios do Rio Grande do Norte, produzindo variáveis resumidas e disponibilizando-a para futuros estudos epidemiológicos. Assim, criar-se-á índices com uma interpretação mais simples, fornecendo informações para a aplicação de recursos em saúde, contribuindo para que os programas, ações e estratégias sejam mais efetivos na promoção da longevidade com qualidade de vida.

\section{Metodologia}

O presente estudo, do tipo ecológico, tem como unidade de observação e análise o estado do Rio Grande do Norte no nível dos seus 167 municípios. Segundo dados do Censo Demográfico realizado pelo IBGE em 2010, a população total deste estado foi de 3.168 .027 habitantes, sendo contabilizados 151.358 $(4,78 \%)$ idosos do sexo masculino e $191.532(6,04 \%)$ do sexo feminino. Assim, a população de idosos corresponde a um total de 342.890 indivíduos com 60 anos ou mais de idade, o que representa 10,82\% da população do Rio Grande do Norte para aquele ano ${ }^{6}$.

Tendo em vista que as variáveis socioeconômicas apresentam efeito nos desfechos de saúde em longo prazo, foram coletadas do passado, referentes ao ano 2000 oriundos de fontes oficiais e confiáveis: Instituto Brasileiro de Geografia e Estatística (IBGE), Programa das Nações Unidas para o Desenvolvimento (PNUD); Instituto de Pesquisa Econômica Aplicada (IPEA) e Departamento de Informática do Sistema Único de Saúde (DATASUS). Partiu-se de um universo de mais de 800 variáveis, das quais 64 foram selecionadas com base na plausibilidade teórica e submetidas a uma análise da distribuição dos dados através de histogramas e boxplots, eliminando aqueles indicadores com uma baixa variabilidade na distribuição dos dados. Diante disso, restaram 20 variáveis relacionadas a Fatores econômicos, Educação, Desenvolvimento Humano, Demografia e Habitação, descritas no quadro 1. 


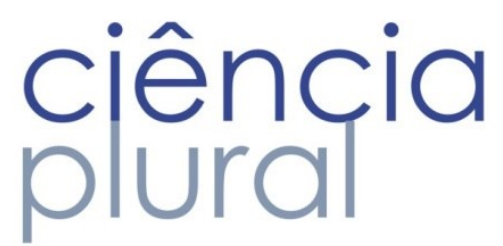

Quadro 1: Caracterização das variáveis independentes contextuais. Natal-RN, 2017.

\begin{tabular}{|c|c|c|}
\hline Nome da Variável & Definição & Fonte \\
\hline Índice de Gini & $\begin{array}{l}\text { Grau de desigualdade existente na distribuição de indivíduos segundo a renda domiciliar per } \\
\text { capita. }\end{array}$ & PNUD-IBGE \\
\hline Índice $L$ de Theil & $\begin{array}{l}\text { Grau de desigualdade segundo o logaritmo da razão entre as médias aritmética e geométrica da } \\
\text { renda domiciliar per capita dos indivíduos. }\end{array}$ & PNUD-IBGE \\
\hline Transferência de renda & $\begin{array}{l}\text { Participação percentual das rendas provenientes de transferências governamentais } \\
\text { (aposentadorias, pensões e programas oficiais de auxílio, como renda mínima, bolsa-escola e } \\
\text { seguro-desemprego) na renda total do município. }\end{array}$ & IPEA \\
\hline Indigentes & $\begin{array}{l}\text { Percentual de pessoas com renda domiciliar per capita inferior a } R \$ 37,75 \text {, equivalentes a } 1 / 4 \text { do } \\
\text { salário mínimo vigente em } 08 / 2000 \text {. }\end{array}$ & IPEA \\
\hline Renda $<1 / 4$ de Salário Mínimo & $\begin{array}{l}\text { Proporção de pessoas com renda menor que } 1 / 4 \text { de salário mínimo, considerando-se o valor de } \\
\text { referência de } 2010(\mathrm{R} \$ 510,00) \text {. }\end{array}$ & DATASUS \\
\hline Analfabetismo & Proporção de analfabetos na população total. & DATASUS \\
\hline Analfabetismo idosos & Proporção de pessoas de 60 anos ou mais analfabetas. & DATASUS \\
\hline $\begin{array}{l}\text { IFDM (Índice Firjan de } \\
\text { Desenvolvimento Municipal) }\end{array}$ & Média simples dos IFDMs de "emprego \& renda", "educação" e "saúde". & IPEA \\
\hline IFDM Saúde & $\begin{array}{l}\text { Média ponderada de indicadores do Ministério da Saúde: número de consultas pré-natal; } \\
\text { óbitos por causa mal definidas e óbitos infantis por causas evitáveis. }\end{array}$ & IPEA \\
\hline IFDM Emprego e Renda & $\begin{array}{l}\text { Média ponderada de indicadores extraídos de duas bases do Ministério do Trabalho: geração de } \\
\text { emprego formal; estoque de emprego formal e salários médios do emprego formal. }\end{array}$ & IPEA \\
\hline IFDM Educação & $\begin{array}{l}\text { Média ponderada de indicadores extraídos de duas bases do Ministério da Educação (MEC): } \\
\text { taxa de matrícula na educação infantil; taxa de abandono; taxa de distorção idade e série; } \\
\text { percentual de docentes com ensino superior; média de horas aula diária e resultado do IDEB. }\end{array}$ & IPEA \\
\hline IDH-M & $\begin{array}{l}\text { Média geométrica dos índices das dimensões Renda, Educação e Longevidade, com pesos } \\
\text { iguais. }\end{array}$ & PNUD \\
\hline Proporção de idosos & Relação calculada entre a população de 60 anos ou mais e a população total. & DATASUS \\
\hline Grau de Urbanização & Proporção calculada da população da área urbana em relação à população total. & PNUD-IBGE \\
\hline Razão de dependência & $\begin{array}{l}\text { Razão entre o segmento etário da população considerado economicamente dependente (os } \\
\text { menores de } 15 \text { anos de idade e os de } 60 \text { e mais anos de idade) e o potencialmente produtivo } \\
\text { (entre } 15 \text { e } 59 \text { anos de idade, multiplicada por } 100 .\end{array}$ & PNUD-IBGE \\
\hline Razão de dependência $>75 \%$ & $\begin{array}{l}\text { Razão entre as pessoas que vivem em domicílios nos quais a razão de dependência é maior do } \\
\text { que } 75 \% \text {, multiplicada por } 100 \text {. }\end{array}$ & IPEA-IBGE \\
\hline Acesso à energia elétrica & $\begin{array}{l}\text { Razão entre a população que vive em domicílios particulares permanentes com iluminação } \\
\text { elétrica e a população total residente em domicílios particulares permanentes multiplicado por } \\
100 .\end{array}$ & PNUD-IBGE \\
\hline Acesso à coleta de lixo & $\begin{array}{l}\text { Razão entre a população que vive em domicílios com coleta de lixo e a população total residente } \\
\text { em domicílios particulares permanentes multiplicado por } 100 \text {. }\end{array}$ & PNUD-IBGE \\
\hline $\begin{array}{l}\text { Acesso à banheiro e água } \\
\text { encanada }\end{array}$ & $\begin{array}{l}\text { Razão entre a população que vive em domicílios particulares permanentes com água encanada } \\
\text { em pelo menos um de seus cômodos e com banheiro exclusivo e a população total residente em } \\
\text { domicílios particulares permanentes } \times 100 \text {. }\end{array}$ & PNUD-IBGE \\
\hline Densidade domiciliar>2 & $\begin{array}{l}\text { Razão entre a população que vive em domicílios particulares permanentes com densidade } \\
\text { domiciliar superior a } 2 \text { e a população total residente em domicílios particulares permanentes } \\
\text { multiplicado por } 100 \text {. }\end{array}$ & PNUD-IBGE \\
\hline
\end{tabular}




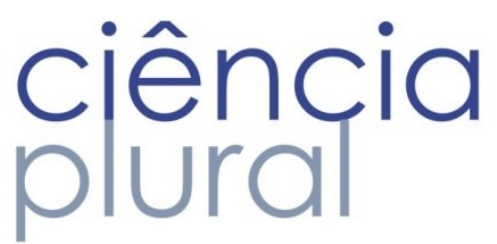

Tais variáveis foram resumidas através da Análise Fatorial $\mathrm{R}$, na qual as mesmas são agrupadas de acordo com as dimensões latentes no seu conjunto e interpretadas por aquilo que representam coletivamente ${ }^{7}$. Tendo em vista que esta se trata de uma análise multivariada, previamente foi realizado um exame dos dados perdidos e atípicos para garantir a sua validade e precisão. Os dados perdidos encontrados se referem a um único município, Jundiá. Diante disso, optou-se pela estimação dos mesmos por intermédio da regressão. Quantos aos outliers, sua identificação se deu sob uma perspectiva multivariada, através da medida de distância de Mahalanobis $\left(D^{2}\right)$.

Atendidas as suposições estatísticas, foi avaliado se as variáveis incluídas na análise fatorial foram suficientemente correlacionadas umas com as outras para produzir fatores representativos. Para tanto, foram calculados a multicolinearidade a partir da matriz de correlações baseada no coeficiente de correlação de Pearson, a medida de adequação da amostra, o teste de esferecidade de Bartlett e a matriz anti-imagem. Com a confirmação da aplicabilidade da técnica estatística fatorial, procedeu-se à extração dos fatores utilizando a Análise de Componentes Principais, já que se tem como objetivo a redução dos dados para obtenção do mínimo de fatores necessários para explicar o máximo de variância representada pelas variáveis originais. 0 número de componentes extraídos foi determinado pelo critério de Kaiser, que informa o quanto cada fator consegue explicar da variância total, e, para facilitar a interpretação dos fatores, fez-se uso do método de rotação ortogonal do tipo Varimax.

Para garantir a confiabilidade do processo de extração dos componentes, foi realizada a validação de conteúdo através de uma segunda análise fatorial. Neste caso, utilizou-se uma subamostra dos municípios do Rio Grande do Norte, com tamanho baseado em uma mínima proporção de casos por variável, sendo aleatoriamente selecionados $72 \%$ dos municípios.

Com vistas a facilitar a compreensão da distribuição dos componentes no território do Rio Grande do Norte, fez-se uso da Análise Espacial de Áreas, sendo apresentadas as representações cartográficas dos mesmos. Esta percepção visual no espaço permite a realização do diagnóstico situacional e definição da escala de prioridades de atenção, contribuindo expressivamente para o planejamento em Saúde, além de permitir testar a plausibilidade teórica dos agrupamentos e fatores formados.

\section{Resultados}

No universo de cerca de 800 variáveis contextuais relacionadas à saúde dos idosos disponíveis em bases de dados secundárias, foi feita a seleção de 20 variáveis de acordo com o fluxograma da figura 1 . 


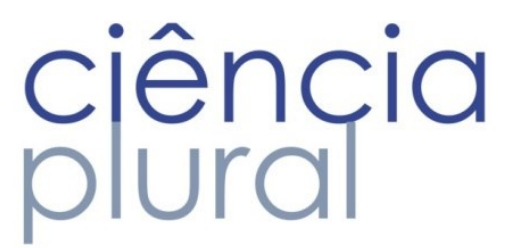

Previamente a tal análise multivariada, procedeu-se à padronização das variáveis para torná-las comparáveis entre si, já que possuem grandezas diferentes. Considerando-se a média igual a zero, o valor das variáveis passa a ser o seu desvio-padrão calculado. Ademais, o tratamento dos dados ausentes e atípicos foi efetuado com vistas a evitar a distorção dos resultados, sendo eliminados os municípios de Galinhos, Serrinha dos Pintos e Vila Flor.

Figura 1: Fluxograma do processo de resumo das variáveis socioeconômicas contextuais do ano 2000 para o Rio Grande do Norte. Natal-RN, 2017.

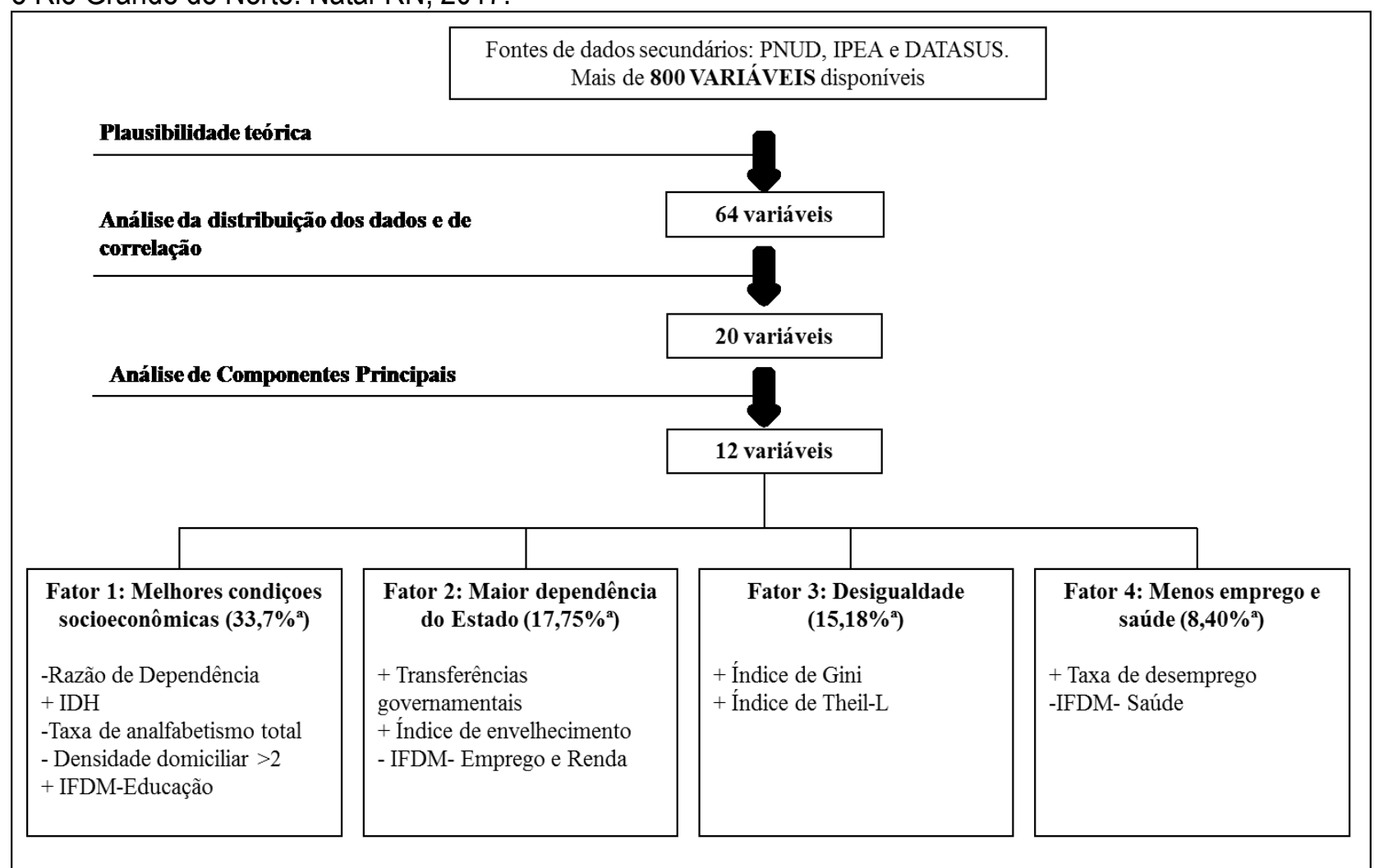

a Percentual da variância total das variáveis incluidas no modelo explicada pelo fator.

As características socioeconômicas dos municípios do Rio Grande do Norte foram sintetizadas por meio da análise fatorial, cuja finalidade primordial foi identificar fatores comuns que traduzissem todas essas informações. Inicialmente, na matriz de correlações, foi identificada a necessidade de exclusão de 13 variáveis por apresentarem correlações significativas e perfeitas com as outras variáveis presentes no modelo. Sendo assim, 12 variáveis na forma de indicadores socioeconômicos participaram da análise fatorial, de fato. Na matriz de correlações das 12 variáveis analisadas, observou-se um número significativo de valores superiores a 0,30 e inferiores a 0,8 , garantindo a aplicabilidade da análise fatorial, uma vez que tais variáveis estão aferindo constructos distintos. 


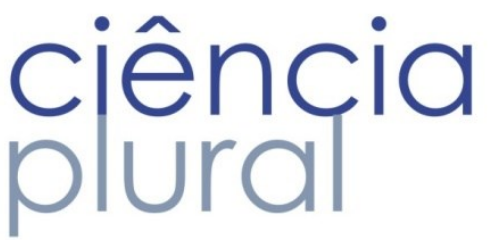

A utilização da técnica estatística adotada se mostrou apropriada também pela avaliação a adequação das variáveis. A estatística KMO (Kaiser-Meyer-Olkin), também considerada uma medida de adequação da amostra, obteve o valor de 0,708, sendo próximo a 1 e indicando a adequação na utilização da técnica. Além disso, o teste de esfericidade de Bartlett apresentou um $p$-valor $(<0,001)$ na faixa de significância estatística, indicando haver correlações significativas entre as variáveis ${ }^{7}$.

A análise da matriz anti-imagem mostrou que apenas as variáveis índice de Theil - $L$ e taxa de desemprego $16+$ apresentaram valores ligeiramente menores que 0,5 na diagonal principal $(0,462$ e 0,471 respectivamente). A permanência dessas variáveis na análise fatorial se justifica pelos valores de comunalidades (tabela 1), que mostraram elevados percentuais de variâncias explicadas pelos componentes extraídos. Além disso, a análise dos valores que se encontram fora da diagonal principal revela uma fraca correlação parcial entre as variáveis do modelo, uma vez que todos os valores estão abaixo de 0,7 . Sendo assim, pode-se concluir que as variáveis selecionadas se correlacionam entre si, sendo pouco influenciadas por outras variáveis não coletadas ou que não participaram da análise fatorial.

Tabela 1: Matriz rotada das cargas fatoriais e comunalidades das variáveis socioeconômicas no estudo dos terminantes socioeconômicos da saúde do idoso no estado do Rio Grande do Norte, Natal-RN, 2017.

\begin{tabular}{llcccc}
\hline Variáveis & Comunalidades & \multicolumn{4}{c}{ Componente } \\
\cline { 3 - 6 } & & $\mathbf{1}$ & $\mathbf{2}$ & $\mathbf{3}$ & $\mathbf{4}$ \\
\hline Índice de Gini & 0,880 & & & 0,912 & \\
Índice de Theil - L & 0,911 & & & 0,932 & \\
Taxa de desemprego 16+ & 0,579 & & & 0,648 \\
Renda de transferências & 0,699 & & 0,836 & \\
governamentais & & & & \\
Razão de dependência & 0,776 & $-0,869$ & & \\
Índice de envelhecimento & 0,816 & 0,570 & 0,649 & \\
IFDM- Educação & 0,599 & 0,759 & & \\
IFDM- Emprego e Renda & 0,573 & & $-0,718$ & \\
IFDM- Saúde & 0,670 & & & \\
IDHM & 0,870 & 0,883 & & \\
\% da população em domicílios & 0,773 & $-0,836$ & & \\
com densidade > 2 & & & & \\
Taxa de analfabetismo total & 0,793 & $-0,788$ & & \\
\hline
\end{tabular}




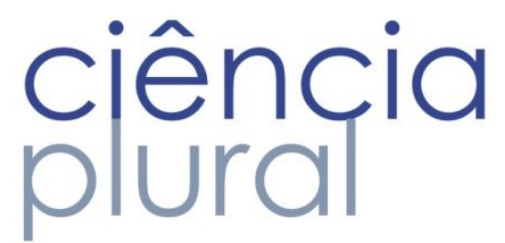

Após a confirmação da aplicabilidade da análise fatorial, partiu-se, de fato, para a análise dos componentes principais. Com base no critério de Kaiser, observa-se que foram retidos quatro componentes que explicaram $74,5 \%$ da variância total das variáveis incluídas no modelo. 0 componente 1 (um) explica sozinho $33,17 \%$ dessa variância, o componente 2 (dois) 17,75\%, enquanto que os componentes 3 (três) e 4 (quatro) explicam $15,18 \%$ e $8.40 \%$, respectivamente.

A matriz rotada das cargas fatoriais apresentada na tabela 1 mostra em destaque os grupos de variáveis pertencentes a cada um dos quatro componentes resultantes da análise em questão. A distribuição das cargas fatoriais é fundamental para a nomenclatura e a interpretação dos componentes extraídos, sendo o fluxograma do processo de resumo das variáveis investigadas e a descrição dos componentes obtidos ilustrados na figura 1 já apresentada. Assim, o componente 1 foi denominado Melhores condições socioeconômicas porque apresenta as características de baixa razão de dependência, alto IDH, menor taxa de analfabetismo total, menor porcentagem de habitações com densidade domiciliar superior a 2 e maior valor do aspecto educação do Índice FIRJAN de Desenvolvimento Municipal (IFDM).

Uma vez que englobou maiores valores de transferências governamentais, de índice de envelhecimento e menores valores do aspecto renda e emprego do IFDM, o componente 2 foi intitulado como Maior dependência do Estado. No componente 3, encontramos maiores valores de variáveis que expressam desigualdade na distribuição de renda (Índice de Gini e Índice de Theil - L) e, portanto, foi denominado Desigualdade. Por fim, o componente 4 agrupou as características de maior taxa de desemprego e menor valor do aspecto saúde do IFDM, sendo nominado como Menos emprego e saúde.

Com vistas a garantir a confiabilidade do processo de extração dos componentes, foi realizada a validação de conteúdo através de uma segunda análise fatorial. Neste caso, utilizou-se uma subamostra dos municípios do Rio Grande do Norte, com tamanho baseado em uma mínima proporção de casos por variável, sendo aleatoriamente selecionados $72 \%$ dos municípios. Aplicada a segunda análise fatorial, 0 critério de Kaiser confirmou que um total de quatro componentes é o número mais adequado, sendo as variáveis distribuídas da mesma forma entre os componentes na matriz rotada pelo método ortogonal Varimax. Destarte, está assegurada a reprodutibilidade e precisão dos componentes que representam os dados contextuais do estado do Rio Grande do Norte e que podem ser utilizados em substituição às variáveis de todo este constructo. 


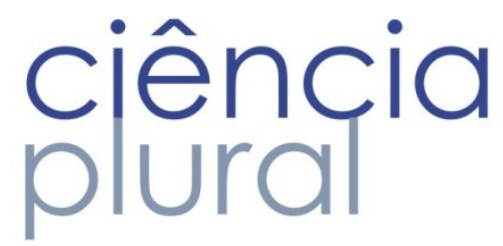

Confirmada a adequação da primeira análise fatorial, a figura 2 apresenta a distribuição espacial dos quatro componentes formados, cujos escores fatoriais foram categorizados em três grupos, segundo dois pontos de corte nos percentis 33 e 67.

Figura 2: Distribuição espacial dos componentes representativos das variáveis socioeconômicas determinantes da saúde da população idosa no estado do Rio Grande do Norte, no ano de 2000. Natal-RN, 2017.

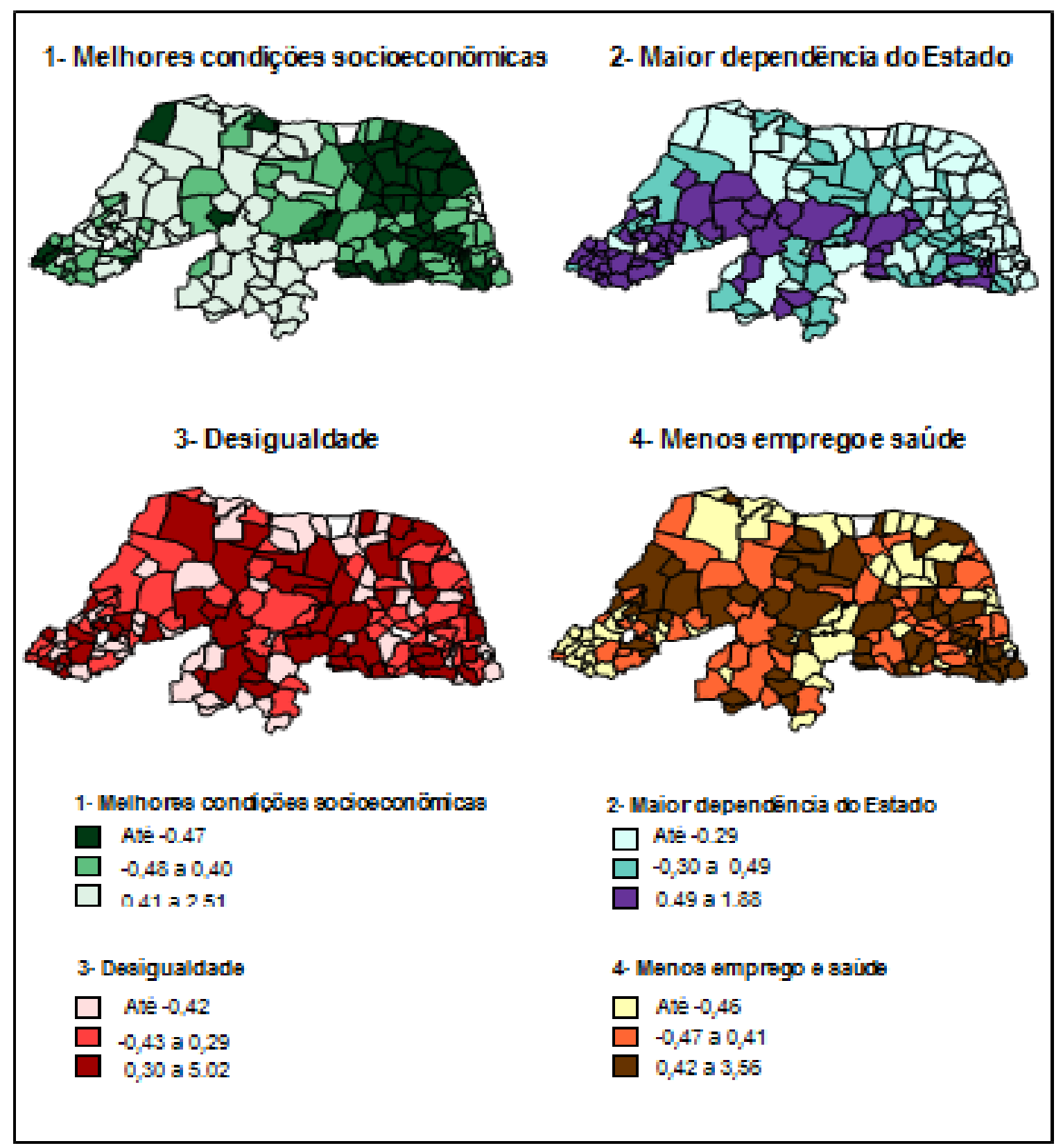




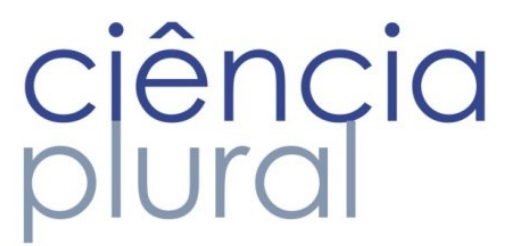

A inspeção visual dos mapas indica que o componente 1, Melhores condições socioeconômicas, divide o estado ao meio. A maior parte dos municípios localizados na porção Oeste do estado apresentam características mais favoráveis de desenvolvimento humano, educação, densidade domiciliar e de dependência da população ativa. Já a região Leste, é fortemente marcada por um perfil desfavorável quanto a este componente, com exceção da capital do estado (Natal) e do município de Parnamirim.

De forma análoga, a distribuição do componente 2, Maior dependência do Estado, apresenta-se com relações de vizinhança, sendo a porção sul, próximo ao limite do estado com a Paraíba, aquela onde há uma intensa concentração de municípios identificados como de maior dependência do Estado. Os municípios menos dependentes são encontrados próximos ao litoral leste potiguar e da microrregião de Mossoró.

Por fim, os componentes 3 e 4, Desigualdade e Menos emprego e saúde, respectivamente, se distribuem de modo mais aleatório ao longo do território estadual. Os maiores índices de desigualdade se apresentam mais densamente nas microrregiões do Seridó Ocidental, Vale do Açu, Borborema Potiguar, Angicos e na região do Alto Oeste. Além da microrregião de Angicos, os menores níveis de emprego e saúde se apresentam com áreas de concentração na Serra de Santana e Chapada do Apodi. Por outro lado, a maior parte do litoral norte do estado exibiu condições mais satisfatórias para os dois componentes em questão.

\section{Discussão}

A análise dos dados do Rio Grande do Norte no âmbito dos seus municípios revelou quatros componentes que resumem o perfil socioeconômico dos mesmos. Apesar de constituir um dos principais eixos para a análise das condições de vida, observou-se a ausência da maior parte das variáveis que mensuram as condições de habitação das famílias dentre tais componentes, excluídas devido à sua alta correlação com outras variáveis, não representando satisfatoriamente o constructo socioeconômico nos municípios investigados.

Além do acesso à iluminação elétrica, as condições de habitação podem ser indicadas pelo saneamento adequado, sendo o abastecimento de água, o esgotamento sanitário e a coleta de lixo fundamentais para uma vida saudável dos moradores e revelam a ação do poder público na gestão das condições de vida da população. No Brasil, 69,4\% dos domicílios urbanos brasileiros tiveram acesso simultâneo aos três referidos serviços de saneamento no ano de 2011, o que configura um quadro 


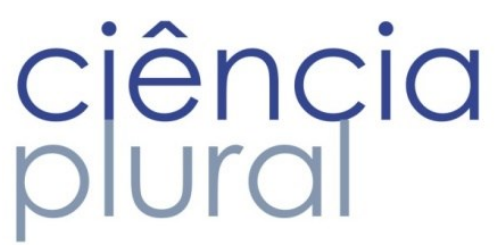

insuficiente, pois 16 milhões de domicílios não apresentavam acesso ao saneamento adequado. Entre as regiões brasileiras, percebe-se uma relevante diferença de proporção de domicílios com serviço de saneamento, que representa $49,9 \%$ no Nordeste e $89,3 \%$ no Sudeste. No Rio grande do Norte, tal percentual é ainda menor (24,4\%), o que justifica a menor influência na representatividade dos fatores socioeconômicos no estado 8 .

O componente 1, nomeado Melhores condições socioeconômicas, configura-se como o de maior relevância para os fatores socioeconômicos dos municípios do Rio Grande do Norte, pois detém o maior percentual de variância explicada e reúne variáveis de várias dimensões: menor razão de dependência demográfica; menor densidade domiciliar; maior Índice de Desenvolvimento Humano (IDH); menores taxas de analfabetismo; e, por fim, maiores valores relacionados à educação. Para compreender este componente multidimensional, nos parágrafos que se seguem são discutidos cada dimensão que o compõe.

A educação apresenta um importante papel positivo como determinante da saúde, sendo sua ausência ou baixa escolaridade relacionada a um menor acesso aos serviços médicos e diagnósticos, levando-os, com maior frequência, a desconhecer sua situação de saúde e, portanto, a presença de determinadas doenças, além de levar a um menor conhecimento quanto à prevenção das mesmas. $\mathrm{Na}$ população idosa, encontramos um percentual de 32\% com um tempo de estudo inferior a um ano, fruto de um deficiente sistema educacional brasileiro no passado que reflete sobre a atual população de 60 anos ou mais de idade com uma média de 3,9 anos de estudo 8,9 .

O indicador educação se mostrou relevante para a formação do componente Melhores condições socioeconômicas, uma vez que, além da taxa de analfabetismo e do IFDM-Educação, foi representado pela variável composta IDH, que contempla taxas de alfabetização e de matrículas. Proposto pelo economista paquistanês MahbubulHaq, o IDH é amplamente utilizado para medir o desenvolvimento de territórios de diversos níveis, desde países até bairros, por exemplo. ${ }^{10}$ Sua inclusão na composição do componente em questão agrega um profundo aporte teórico, dado que ele abrange muitas informações dos determinantes sociais, relacionadas às dimensões longevidade e renda, além da já citada educação. Ademais, em concordância com todas as variáveis do componente, os maiores valores de IDH no mesmo ratifica a característica de vantagem socioeconômica.

A variável densidade domiciliar carrega informações a respeito das condições de moradia dos indivíduos, mais especificamente quanto ao tamanho das residências e à capacidade de abrigar as famílias adequadamente ${ }^{11}$. Assim, o nível de densidade domiciliar reduzido no componente 1 demonstra a 


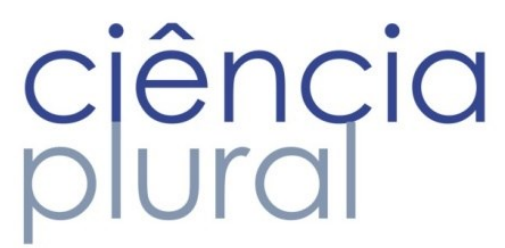

expressiva parcela de moradias com condições favoráveis de privacidade e conforto. Ademais, tal variável pode ser considerada um proxy da renda das famílias e, portanto, um objetivo e claro indicador socioeconômico.

A característica de menor razão de dependência demográfica encontrada no componente Melhores condições socioeconômicas é um fator que favorece as políticas previdenciárias e se configura em uma oportunidade de crescimento econômico devido à maior parcela de pessoas em idade ativa. Diante disso, é importante que os municípios com altos níveis do referido componente mantenham um suficiente crescimento da economia e adequada regulação do mercado de trabalho, para que não haja uma alta proporção de desempregados e empregados na informalidade. Assim, é preciso aproveitar a atual oportunidade demográfica de relevante população em idade ativa para se obter uma estrutura na sociedade que atenda ao progressivo aumento do peso da população dependente, causado pelo incremento do número de idosos ${ }^{12}$.

A partir da distribuição espacial do componente 1, que representa um único do modelo com características socioeconômicas positivas, pode-se observar que os determinantes mais favoráveis à saúde estão presentes nas microrregiões do Seridó, Alto e Médio Oeste, assim como também a do Vale do Açu. Dessas regiões, a mais populosa é a do Seridó, que representa apenas $11 \%$ da população Estadual, o que expõe o fato de que a parcela populacional que usufrui de fatores que favorecem a preservação da qualidade de vida é ainda muito pequena. ${ }^{6}$ Por outro lado, as políticas públicas devem concentrar uma maior atenção nos municípios próximos ao litoral nordeste, com exceção da capital Natal e da cidade de Parnamirim, que apesar de ser uma região desenvolvida, apresentou condições socioeconômicas da população mais desfavoráveis.

O componente 3, Desigualdade, expressa grande importância para a compreensão do contexto socioeconômico local, pois a riqueza de um município nem sempre está em concordância com as condições de vida da sua população, o que pode ser explicado por uma elevada concentração de renda. A disparidade regional de renda é claramente observada na população idosa brasileira, com $68 \%$ dos idosos do Nordeste residindo em domicílios com renda familiar per capita menor que um salário mínimo, ao passo que este percentual está abaixo de 35\% no Sul e no Sudeste. Soma-se à desigualdade de renda, a desigualdade de gênero, que desfavorece a parcela feminina, maioria na população idosa ( $55,7 \%$ desta). Há um histórico de escassa participação desta no mercado de trabalho, com benefícios mínimos de aposentadoria8,13. 


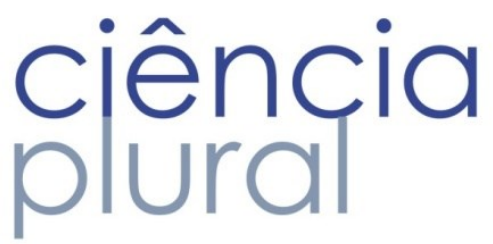

Nota-se uma distribuição bastante heterogênea da Desigualdade, com presença de índices médios e altos por todo 0 território potiguar. Observa-se uma grande concentração de municípios com menor desigualdade nas regiões do Seridó e do Vale do Açu, porém valores altos de desigualdade em relação ao estado são também encontrados de forma relevante. Por essa disposição se repetir por toda a extensão do estado, percebe-se uma necessidade de implementação de medidas para minimizar a desigualdade também em todo o estado, de forma a garantir a equidade socioeconômica e em saúde.

Agrupado no quarto componente, Menos emprego e saúde, o desemprego é um dos maiores problemas sociais da humanidade ${ }^{14}$. Segundo Blanc, Katz e Yelin em 199415, apenas a situação de estar trabalhando se constitui um potente e independente fator preditivo para uma maior sobrevida. Aliado ao emprego, a qualificação ocupacional do indivíduo exerce influência sobre a saúde, o que é explicado pela relação direta com a escolaridade, renda, autoestima e condições de vida de um modo geral. No entanto, pouco se sabe ainda sobre o efeito da permanência no mercado de trabalho em idades mais avançadas ${ }^{16}$.

Como determinante socioeconômico em nível ecológico, acredita-se que a taxa de desemprego revela características de desenvolvimento municipal, interferindo nas condições de vida dos indivíduos ativos e seus dependentes, como os idosos em algumas famílias. O quarto componente, Menos emprego e saúde, demonstra claramente a relação inversa do desemprego com a saúde, representada pelo IFDM-Saúde. Este índice retrata a Atenção Básica, cujos indicadores são de competência municipal: proporção de mortes por causas mal definidas, internações sensiveis à Atenção Básica, percentual de gestantes com mais de seis consultas pré-natal e taxa de óbitos infantis por causas evitáveis ${ }^{17}$.

Assim, observa-se que a maior parte dos municípios das microrregiões Chapada do Apodi, Litoral Sul e Angicos apresentam uma deficiência importante na Atenção Básica. Faz-se necessária uma atenção maior e mais urgente do auxilio aos gestores municipais para a qualificação dos serviços básicos de saúde prestados nos mesmos e fortalecimento desse nível de atenção no sistema público de saúde, aliada à necessidade de maior provimento de oportunidades de trabalho.

O emprego também obteve um papel importante no componente 2 - Maior dependência do Estado, desta vez acompanhado da renda, obtendo valores mais desfavoráveis em grande parte dos municípios localizados ao sul do estado. Medido em função do IFDM- Emprego e renda, este indicador expressa a geração de emprego formal e a capacidade de absorção da mão-de-obra local, acompanhado da geração de renda e sua distribuição no mercado de trabalho do município ${ }^{17}$. 


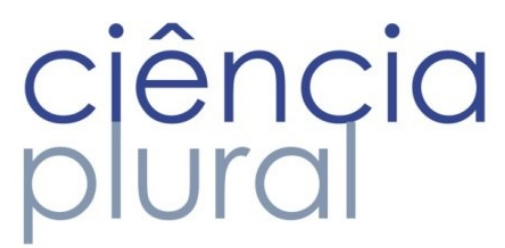

A renda constitui uma das variáveis mais utilizadas nas análises em saúde, desde em nível de indivíduos, de grupos populacionais, até países. Seu efeito já está bem estabelecido na literatura, revelando uma relação causal com 0 aparecimento de doenças e morte precoce ${ }^{18}$. Nesse sentido, destaca-se a população idosa com grandes demandas de cuidados em saúde e forte exclusão social, na qual a menor renda pode conduzir a uma menor utilização dos serviços de saúde, refletindo em uma condição de saúde e função física menos favoráve|19,20.

Diante das demandas sociais, ocorreram profundos avanços nas políticas públicas no decorrer dos últimos anos, promovendo ações e estratégias que visam dar respostas aos principais problemas emergentes ${ }^{21}$. Em relação aos idosos, as garantias de benefícios de, pelo menos, um salário mínimo para aqueles carentes do país, permitiu que os mesmos conseguissem uma situação de rendimento familiar mais favorável que crianças, adolescentes e jovens atualmente ${ }^{8}$. 0 segundo componente contempla estas políticas de assistência social e previdenciárias, que detêm uma participação percentual relevante na renda total dos municípios com maiores valores do componente $2 \mathrm{e}$, apesar de demonstrarem um importante apoio financeiro do governo para a população, significam uma maior dependência do Estado.

Com o maior índice de envelhecimento também presente no componente em questão, a situação de dependência financeira se agrava. Calculado a partir do número de pessoas de 60 ou mais anos de idade para cada 100 pessoas menores de 15 anos (componentes etários extremos da população), este índice demonstra que a população economicamente ativa tende a diminuir, crescendo a proporção de idosos, dependente desta população que gera renda22. Apesar de constituir um ponto positivo per si, devido à conquista do maior envelhecimento da população, a situação elevada deste índice suscita o planejamento de políticas públicas que estejam em consonância com o novo perfil populacional. Como demonstrado neste índice, as questões de trabalho e renda se apresentam como uma das demandas para ser repensada e adaptada de modo que se garanta a sustentabilidade das transferências governamentais pela população economicamente ativa.

Como limitação, tem-se que estes indicadores compostos têm utilidade maior para analisar seu efeito sobre as desigualdades em saúde em geral, em nível ecológico, sem informar quais são os fatores relacionados ao mecanismo de produção das desigualdades ${ }^{23}$. Para atender a este último objetivo mais específico, as variáveis originais são mais adequadas, tendo sido observado que, dentre as variáveis contextuais utilizadas, o IFDM detém uma grande importância como indicador socioeconômico, sendo sua alta representação validada pela presença das suas categorias em três dos quatro componentes formados. 


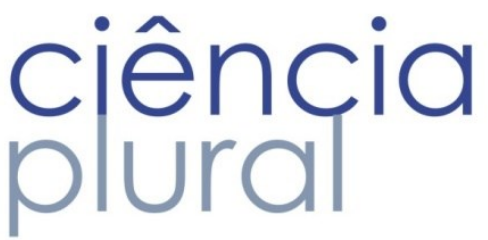

Além da identificação dos pontos críticos e utilização nas análises do efeito do constructo contextual sobre as desigualdades em saúde em idosos, os componentes obtidos podem ser inseridos em análises multivariadas em estudos que os determinam como fatores de confusão, para que sejam controlados. Ademais, seu uso se justifica também nos casos em que todos os indicadores potenciais estão correlacionados ou quando não há uma plausibilidade teórica para eleger um indicador simples como prioritário em relação a outros 23,24 .

\section{Conclusões}

Os componentes criados (Melhores condições socioeconômicas, Maior dependência do Estado, Desigualdade e Menos emprego e saúde) resumiram e englobaram diversas dimensões contextuais, de forma conjunta, obtendo-se uma maior aproximação da real complexidade que envolve o contexto socioeconômico, sob a perspectiva da população idosa. Deste modo, este trabalho contribui para o estudo das desigualdades em saúde de idosos no estado do Rio Grande do Norte, a partir de uma análise objetiva que destacou, em geral, os municípios nas microrregiões Borborema Potiguar, Angicos e Agreste Potiguar como aqueles em situação mais desfavorável e que necessitam, portanto, de uma maior atenção por parte das políticas públicas. Portanto, os índices produzidos fornecem subsídios para o planejamento de políticas públicas, bem como podem ser utilizados em outros estudos como variáveis que representam o nível socioeconômico contextual para os municípios do Rio Grande do Norte.

\section{Referências}

1. Veras R. Envelhecimento populacional contemporâneo: demandas, desafios e inovações. Rev Saúde Pública. 2009;43(3): 548-554.

2. Santos AMA, Sousa EA. Uma análise comparativa entre os indicadores socioeconômicos da região Nordeste e do Brasil. Revista Economia política do desenvolvimento. RevEconPolit. 2010;3(8): 43-72.

3. Santos M, Silveira ML. O Brasil: território e sociedade no início do século XXI. 12a ed. Rio de Janeiro, RJ: Record; 2008.

4. Borrell C, Artazcoz L. Las políticas para disminuirlas desigualdades ensalud. GacSanit. 2008;22(5): 465-473.

5. Oliveira TC, Medeiros WR, Freitas YNL, Lima KC. Socioeconomic factors and the elderly mortality profile in a poor brazilian state: An ecological study comparing two distinct Age Groups. OALib. 2015;2(1271): $1-8$. 


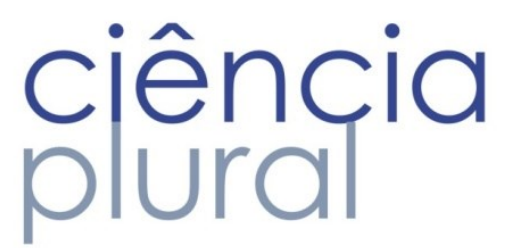

6. BrasiL. Ministério da Saúde. Informações de Saúde[internet ] [capturado em 2013]. Disponível em: http://www2.datasus.gov.br/DATASUS/index.php?area=0206.

7. Fávero LPL, Belfiore, PP, Silva, FL, Chan, BL. Análise de dados: modelagem multivariada para tomada de decisões. Rio de Janeiro, Elsevier: 2009.

8. Instituto Brasileiro de Geografia e Estatística (IBGE). Síntese de indicadores sociais: uma análise das condições de vida da população brasileira. Rio de Janeiro: IBGE, 2013.

9. Barros MBA, Francisco PMSB, Zanchetta LM, César, CLG. Tendências das desisgualdades sociais e demográficas na prevalência de doenças crônicas no Brasil, PNAD: 2003-2008. Ciênc Saúde Coletiva. 2011;16(9): 3755-3768.

10. Abreu MVS, Oliveira JC, Andrade VDA, Meira AD. Proposta metodológica para o cálculo e análise espacial do IDH intraurbano de Viçosa - MG. RevBrasEstud Popul. 2011;28(1): 169-186.

11. Givisiez GHN, Oliveira EL. Privacidade intradomiciliar: um estudo sobre as necessidades de ampliações em residências. RevBrasEstud Popul. 2013;30(1): 199-223.

12. Brito F. Transição demográfica e desigualdades sociais no Brasil. RevBrasEstud Popul. 2008;25(1): 526.

13. Geib LTC. Determinantes sociais da saúde do idoso. Ciênc Saúde Coletiva. 2012;17(1): 123-133.

14. Oliveira, EA. A política de emprego no Brasil: o caminho da flexinsegurança.ServSoc Soc.2012;111: 493-508.

15. Blanc PD, Katz P, Yelin, E.Mortality risk among elderly workers. American Journalof Industrial Medicine. 1994;26: 543-547.

16. Giatti L, Barreto SM.Saúde, trabalho e envelhecimento no Brasil. Cad Saúde Pública. 2003;19(3): 759771.

17. Índice Firjan de desenvolvimento municipal[internet]. Rio de Janeiro. [capturado em21 out 2015]. Disponível em:http://www.firjan.org.br/ifdm/downloads

18. Musgrove P. The dethronement of income as a cause of health: an essay. RevBra. SaudeMater Infant.2007;7(4): 461-466.

19. Cesar JA, Oliveira Filho JA, Bess G, Cegielka R, Machado J, Gonçalves TS, 2008. Perfil dos idosos residentes em dois municípios pobres das regiões Norte e Nordeste do Brasil: resultados de estudo transversal de base populacional. CadSaúdePública. 24(8), 1835-1845.

20. Oliveira TC, Silva DA, Freitas YNL, Silva RL, Pegado CPC, Lima KC. Socio-demographic factors and oral health conditions in the elderly: A population-based study. ArchGerontolGeriatr.2013;57(3): 389-97.

21. Ruíz AKLB, Godoi TC, Franco, LFR. Processo do envelhecimento e possíveis implicações das políticas públicas de saúde. Pensamento Plural: RevCientUNIFAE. 2010;4(2): 43-48. 


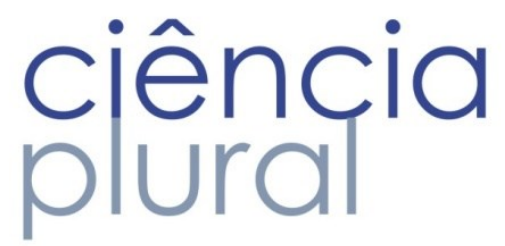

22. Closs VE, Schwanke CHA. A evolução do índice de envelhecimento no Brasil, nas suas regiões e unidades federativas no período de 1970 a 2010. RevBrasGeriatrGerontol. 2012;15(3): 443-458.

23. Domínguez-Berjón FM, Borrell C, Cano-Serral G, Esmola S, Nolasco A, Pasarin MI, et al. Construcción de un índice de privación a partir de datoscensalesen grandes ciudadesespañolas (Proyecto MEDEA). GacSanit. 2008;22(3):179-87.

24. Domínguez-Berjón MF, Rodríguez-Sanz M, Marí-Dell'Olmo M,Esnaolas S, Prieto-Salceda D, Duque I et al. Uso de indicadores socioeconómicos del área de residenciaenlainvestigación epidemiológica: experienciaenEspaña y oportunidades de avance. GacSanit. 2014;28(5):418-425. 